\title{
MS27-P04 | Endohedral Metallofullerene Crystals: Playing With Disorders
}

Liu, Fupin (IFW-Dresden, Dresden, GER); Popov, Alexey A. (IFW-Dresden, Dresden, GER)

Endohedral metallofullerenes (EMFs) are fullerenes encapsulating metal ions or clusters [1]. These molecules are interesting for their characteristic structures and potential applications in the fields of energy conversion, biomedicine, and molecular magnets. One of the great challenges for studying these molecules is the structural elucidation. Because of the round shape of the molecule, crystals of EMF frequently suffered severe disorder. Cocrystallization with structural complementary molecules such as nickel octaethylporphyrin could reduce the disorder $[2,3,4]$, otherwise, functionalizing the fullerene cages with functional groups may also reduce the disorder by restraining the rotation of the fullerenes in the crystal lattice [5]. The dynamics of the encapsulated species is a very interesting topic when we froze the movement of the fullerene cage. Recently, we observed the movement of the two encapsulated Dy ions in $C_{80}$ fullerene cage via variable temperature X-ray diffraction studies [6]. In this contribution, the disorder problem frequently encountered in EMF crystals as well as the successful observation of the movement of encapsulated two Dy ions will be discussed.

1. A. A. Popov et al., Chem. Rev. 2013, 113, 5989-6113.

2. F. Liu et al., J. Am. Chem. Soc. 2016, 138, 14764-14771.

3. F. Liu et al., Angew. Chem. 2017, 129, 1856-1860.

4. D. S. Krylov et al., Phys. Chem. Chem. Phys. 2018, 20, 11656-11672.

5. F. Liu et al., Nat. Commun. 2017, 8, 16098.

6. F. Liu et al., Nat. Commun. 2019, 10, 571. 